\title{
Erratum to: Hygro-thermo-mechanical bending and vibration analysis of the CNTRC doubly curved nanoshells with thickness stretching based on nonlocal strain gradient theory
}

\author{
Hao Wei ${ }^{1, \mathrm{a}}$, Reza Mohammadi ${ }^{2, \mathrm{~b}}$ \\ ${ }^{1}$ Suzhou Zhongheng Tong Road and Bridge Co. Ltd., Suzhou 215124, China \\ 2 Department of Mechanical Engineering, Tarbiat Modares University, Tehran, Iran
}

(C) The Author(s), under exclusive licence to Società Italiana di Fisica and Springer-Verlag GmbH Germany, part of Springer Nature 2021

Erratum to: Eur. Phys. J. Plus (2021) 136:326

https://doi.org/10.1140/epjp/s13360-021-01296-0

In the original publication of the article, unfortunately the corresponding author was wrong. The original article has been corrected.

The original article can be found online at https://doi.org/10.1140/epjp/s13360-021-01296-0.

\footnotetext{
a e-mail: 15295030686@163.com (corresponding author)

b e-mail: mohammadireza456@yahoo.com
} 